\title{
THE NUMERICAL RANGE OF AN UNBOUNDED OPERATOR
}

\author{
M. J. CRABB
}

Abstract. The numerical range of an unbounded linear operator on a complex Banach space is the whole complex plane.

Let $X$ denote a Banach space over $\mathbf{C}, X^{\prime}$ the dual space of $X, S$ $=\{x \in X:\|x\|=1\}$, and let $T$ be an unbounded operator defined on the whole of $X$. Given $x \in S$, let $V(T, x)=\left\{f(T x): f \in X^{\prime},\|f\|=f(x)=1\right\}$. The numerical range $V(T)$ is defined by

$$
V(T)=\cup\{V(T, x): x \in S\} .
$$

J. R. Giles and G. Joseph [2] prove that the semi-inner-product numerical range $W(T)$ has a certain density property, and $\mathrm{B}$. Bollobas and $\mathrm{S}$. Eldridge (preprint) prove that $W(T)$ is dense in C. These imply the corresponding results for $V(T)$.

TheOREM. $V(T)=\mathrm{C}$.

We use the following slight extension of Theorem 1 of [1].

Lemma. Let $x, y \in X$, and operator $R$ be defined on $\lim (x, y)$. Suppose that $\|x+R y\|<\|x\|-(8\|R x\|\|y\|)^{1 / 2}$. Then $\cup\{V(R, z): z \in S \cap \lim (x, y)\}$ contains 0 as an interior point.

Proof. There is a continuous linear operator $R_{1}$ on $X$ such that $R=R_{1}$ on $\lim (x, y)$. The proof in [1] shows that 0 is an interior point of $\cup\left\{V\left(R_{1}, z\right): z\right.$ $\in S \cap \lim (x, y)\}$ which gives the result.

Proof of Theorem. As in [2], there is a sequence $\left(x_{n}\right)$ in $X$ such that $x_{n} \rightarrow 0$ and $T x_{n} \rightarrow-x \neq 0$. Choose $x_{n}$ such that

$$
\left\|x+T x_{n}\right\|<\|x\|-\left(8\|T x\|\left\|x_{n}\right\|\right)^{1 / 2} \text {. }
$$

By the Lemma $0 \in V(T)$. For any $\alpha \in \mathrm{C}, T-\alpha I$ is unbounded, so 0 $\in V(T-\alpha I)$. Hence $\alpha \in V(T)$.

The Lemma implies that, for $T$ defined on a subspace of $X$ with $V(T) \subset \mathbf{R}_{3}$ where we take $V(T)=\cup\{V(T, x): x \in S, T x$ defined $\}$, we have $\|T x\|^{2}$ $\leqslant M\|x\|\left\|T^{2} x\right\|$ with $M=8$. A result of Hille [3] implies that this holds with $M=2$, and an example of Kolmogorov [4] (differentiation on $L_{\infty}(\mathbf{R})$ ) shows that 2 is the best constant.

Received by the editors March 12, 1975.

AMS (MOS) subject classifications (1970). Primary 47A 10.

Key words and phrases. Numerical range, unbounded linear operator.

O American Mathematical Society 1976 


\section{REFERENCES}

1. M. J. Crabb and A. M. Sinclair, On the boundary of the spatial numerical range, Bull. London Math. Soc. 4 (1972), 17-19. MR 46 \#7929.

2. J. R. Giles and G. Joseph, The numerical ranges of unbounded operators, Bull. Austral. Math. Soc. 11 (1974), 31-36.

3. E. Hille, Generalizations of Landau's inequality to linear operators, Conf. on Linear Operators and Approximation, Oberwolfach, August 1971.

4. A. N. Kolmogorov, On inequalities between the upper bounds of the successive derivatives of an arbitrary function on an infinite interval, Učen. Zap. Moskov. Gos. Univ. Matematika 30 (1939), 3-13; English transl., Amer. Math. Soc. Transl. (1) 2 (1962), 233-243. MR 1, 298.

Department of Mathematics, The University, Glasgow G12 8QW, Scotland 